Diffraction of a classical electromagnetic wave by a thin periodic slab: a rigorous approach

This content has been downloaded from IOPscience. Please scroll down to see the full text. 1978 J. Phys. A: Math. Gen. 111855

(http://iopscience.iop.org/0305-4470/11/9/019)

View the table of contents for this issue, or go to the journal homepage for more

Download details:

IP Address: 147.96.14.15

This content was downloaded on 04/02/2014 at 16:43

Please note that terms and conditions apply. 


\title{
Diffraction of a classical electromagnetic wave by a thin periodic slab: a rigorous approach
}

\author{
R F Alvarez-Estrada and M L Calvo \\ Departamento de Física Teórica and Departamento de Optica y Estructura de la Materia, \\ Facultad de Ciencias Físicas, Universidad-Complutense, Madrid, Spain
}

Received 11 January 1978, in final form 30 March 1978

\begin{abstract}
The interaction and diffraction of a classical electromagnetic wave by a thin slab characterised by a periodic dielectric permeability is studied rigorously, thereby completing previously existing studies. New integral equations for the induction vector, in terms of a suitable Green function, are presented, and a condition which ensures the convergence of their iterations is obtained. A bound for the error of the usual Debye-BornRayleigh-Gans approximation to the diffraction amplitudes is given.
\end{abstract}

\section{Introduction}

The interaction and diffraction of a classical electromagnetic wave by a slab characterised by a periodic dielectric permeability is an interesting physical process which appears in several different situations: (i) diffraction of $x$-rays by thin crystals (Batterman and Cole 1964, Ewald 1965, James 1963, Landau and Lifchitz 1969, Slater 1967, Zachariasen 1945); (ii) diffraction of light by ultrasonic waves (Born and Wolf 1965); (iii) diffraction of light by a hologram (Vienot et al 1971, and references therein). From a theoretical standpoint, the subject is a well defined branch of electromagnetic scattering theory, but in order to achieve an understanding of it comparable to that already reached about other scattering phenomena, several interesting and open problems should be solved.

Two of them are: $(a)$ to set up a proper treatment of the electromagnetic field through integral equations (the most natural framework for dealing with scattering problems), which take into account its transverse (divergenceless) nature and, at the same time, its interaction with a periodic structure; $(b)$ to control mathematically the convergence of those integral equations and the diffraction amplitudes.

This paper will provide solutions to the above problems $(a)$ and $(b)$. Sections 2 and 3 will present the relevant Green functions, and new and suitable integral equations for the induction vector, as well as a condition which implies the convergence of the iterations of those integral equations. In $\$ 4$, the diffraction amplitudes are discussed, and a bound for the error of the customary Debye-BornRayleigh-Gans approximation is given. Estimates and applications are discussed in $\S 5$. 


\section{Formulation of the problem}

We consider two vectors $\bar{a}_{1}=\left(a_{1}, 0\right)$ and $\bar{a}_{2}=\left(a_{2}, 0\right), a_{1}$ and $a_{2}$ being two-dimensional vectors with $a_{1}, a_{2} \neq 0$, and regard the direction orthogonal to the $a_{1}-a_{2}$ plane as the $z$ axis. The position of any point in space is determined by $\bar{x}=(l, z), l$ being twodimensional. Inside a macroscopically, homogeneous, isotropic and stationary infinite medium, there is a slab of thickness $a$, which occupies the region $-a / 2<z<+a / 2$ and has an infinite extension in the $\overline{\boldsymbol{a}}_{1}-\overline{\boldsymbol{a}}_{2}$ plane.

A classical monochromatic electromagnetic wave comes in from $z=-\infty$ in the remote past, with wavevector $\overline{\boldsymbol{K}}=\left(\boldsymbol{K}, K_{3}\right)=\left(K_{1}, K_{2}, K_{3}\right)$ and polarisation index $\lambda(\lambda$ taking only two values), and approaches the slab.

In the medium, which has constant dielectric permeability $\epsilon_{0}$, vanishing macroscopic conductivity and magnetic permeability equal to that of the vacuum, we shall represent the wave for later convenience by the induction vector $\overline{\boldsymbol{D}}_{0}(\overline{\boldsymbol{x}}, \overline{\boldsymbol{K}}, \lambda)=$ $\overline{\boldsymbol{D}}_{0}(\overline{\boldsymbol{K}}, \lambda) \exp (\mathrm{i} \overline{\boldsymbol{K}} \cdot \overline{\boldsymbol{x}})$ (all time-dependent factors being factored out). Here, $\overline{\boldsymbol{D}}_{0}(\overline{\boldsymbol{K}}, \lambda)$ is a complex polarisation vector such that $\overline{\boldsymbol{K}} \cdot \overline{\boldsymbol{D}}_{0}(\overline{\boldsymbol{K}}, \lambda)=0$ and $(\alpha, \beta=1,2,3)$

$$
\sum_{\lambda} D_{0}(\overline{\boldsymbol{K}}, \lambda)_{\alpha} D_{0}^{*}(\overline{\boldsymbol{K}}, \lambda)_{\beta}=\delta_{\alpha \beta}-\frac{K_{\alpha} K_{\beta}}{K^{2}}, \quad K=\left(\boldsymbol{K}^{2}+K_{3}^{2}\right)^{1 / 2}
$$

By assumption, the slab has zero electric charge and conductivity, magnetic permeability equal to that of the vacuum and dielectric permeability (in general, frequency-dependent)

$$
\epsilon=\epsilon(l, z) \neq \epsilon_{0}, \quad-a / 2<z<+a / 2
$$

fulfilling $\epsilon\left(l+n_{1} a_{1}+n_{2} a_{2}, z\right)=\epsilon(l, z)$ for any $n_{1}, n_{2}=0, \pm 1, \pm 2, \ldots$

The interaction and diffraction, transmission and reflection of the full electromagnetic wave will be described by the divergenceless complex induction vector, $\overline{\boldsymbol{D}}(\overline{\boldsymbol{x}}, \overline{\boldsymbol{K}}, \lambda)$.

Maxwell's equations and the previous assumptions lead easily to the wave equation (Gaussian units being used)

$$
\left(\Delta_{\bar{x}}+K^{2}\right) \overline{\boldsymbol{D}}(\overline{\boldsymbol{x}}, \overline{\boldsymbol{K}}, \lambda)= \begin{cases}0, & \overline{\boldsymbol{x}} \text { outside the slab } \\ \overline{\boldsymbol{j}}(\overline{\boldsymbol{x}}), & \overline{\boldsymbol{x}} \text { inside the slab }\end{cases}
$$

where

$$
\bar{j}(\bar{x})=K^{2}\left(1-\frac{\epsilon}{\epsilon_{0}}\right) \bar{D}+\epsilon \bar{\nabla} \times\left[\left(\bar{\nabla} \frac{1}{\epsilon}\right) \times \bar{D}\right]-(\bar{\nabla} \ln \epsilon) \times(\bar{\nabla} \times \bar{D}) .
$$

One has $\bar{\nabla} \cdot \bar{j}=0$. In order to determine $\overline{\boldsymbol{D}}(\overline{\boldsymbol{x}}, \overline{\boldsymbol{K}}, \lambda)$, it will be quite convenient, using standard techniques, to combine the above wave equation and the information on the incoming wave into the following integral equation for any $\overline{\boldsymbol{x}}$ :

$\overline{\boldsymbol{D}}(\boldsymbol{l}, z, \overline{\boldsymbol{K}}, \lambda)=\overline{\boldsymbol{D}}_{0}(\overline{\boldsymbol{x}}, \overline{\boldsymbol{K}}, \lambda)+\int_{-\infty}^{+\infty} \mathrm{d}^{2} \boldsymbol{l}^{\prime} \int_{-a / 2}^{+a / 2} \mathrm{~d} z^{\prime} \cdot \mathbf{G}^{\mathrm{T}}\left(\boldsymbol{l}-\boldsymbol{l}^{\prime}, z-z^{\prime}\right) \overline{\boldsymbol{j}}\left(\boldsymbol{l}^{\prime}, z^{\prime}\right)$.

We have used the following tensor Green function $\left(\mathbf{G}^{\mathrm{T}}=\left(G_{\alpha \beta}^{\mathrm{T}}\right), \alpha, \beta=1,2,3\right)$ (Calvo 
and Durán 1975):

$$
\begin{gathered}
G_{\alpha \beta}^{\mathrm{T}}(\overline{\boldsymbol{x}})=\frac{1}{(2 \pi)^{3} K^{2}} \int \mathrm{d}^{3} \overline{\boldsymbol{K}}^{\prime}\left(\frac{K_{\alpha}^{\prime} K_{\beta}^{\prime}-K^{2} \delta_{\alpha \beta}}{K^{\prime 2}-\left(K^{2}+\mathrm{i} \eta\right)}-\frac{K_{\alpha}^{\prime} K_{\beta}^{\prime}}{K^{\prime 2}}\right) \exp \left(\mathrm{i} \overline{\boldsymbol{K}}^{\prime} \cdot \overline{\boldsymbol{x}}\right) \quad \eta>0, \eta \rightarrow 0 \\
=-\frac{\exp (\mathrm{i} K|\overline{\boldsymbol{x}}|)}{4 \pi|\overline{\boldsymbol{x}}|} \delta_{\alpha \beta}+\frac{1}{4 \pi K^{2}} \frac{\partial^{2}}{\partial x_{\alpha} \partial x_{\beta}}\left(\frac{1-\exp (\mathrm{i} K|\overline{\boldsymbol{x}}|)}{|\overline{\boldsymbol{x}}|}\right)
\end{gathered}
$$

instead of the usual Green function (Newton 1966)

$$
\Gamma_{\alpha \beta}(\overline{\boldsymbol{x}})=-\left(\delta_{\alpha \beta}+\frac{1}{K^{2}} \frac{\partial^{2}}{\partial x_{\alpha} \partial x_{\beta}}\right) \frac{\exp (\mathrm{i} K|\overline{\boldsymbol{x}}|)}{4 \pi|\overline{\boldsymbol{x}}|} .
$$

As $|\overline{\boldsymbol{x}}| \rightarrow 0$,

$$
G_{\alpha \beta}^{\mathrm{T}}(\bar{x}) \simeq-\frac{1}{8 \pi|\bar{x}|}\left(\delta_{\alpha \beta}+\frac{x_{\alpha} x_{\beta}}{|\bar{x}|^{2}}\right)
$$

while $\Gamma_{\alpha \beta}(\overline{\boldsymbol{x}})$ diverges as $1 /|\overline{\boldsymbol{x}}|^{3}$. On the other hand, $G_{\alpha \beta}^{\mathrm{T}}$ is divergenceless throughout all space, whereas $\Gamma_{\alpha \beta}$ is not (see Calvo and Durán 1975). These properties will make $G_{\alpha \beta}^{\mathbf{T}}$ far more convenient than $\Gamma_{\alpha \beta}$ regarding the determination of $\overline{\boldsymbol{D}}(\overline{\boldsymbol{x}}, \overline{\boldsymbol{K}}, \lambda)$, as we shall see. To start with, the use of $G_{\alpha \beta}^{\mathrm{T}}$ makes equation (2.4) automatically consistent with $\bar{\nabla} \cdot \bar{D}=0$, while that of $\Gamma_{\alpha \beta}$, in a similar integral equation, would require further work in order to verify such a condition. Another more important advantage of $G_{\alpha \beta}^{\mathrm{T}}$ will show up when we try to set up a convergent iterative determination of $\overline{\boldsymbol{D}}(\overline{\boldsymbol{x}}, \overline{\boldsymbol{K}}, \lambda)$.

Although equation (2.4) can be formally iterated as it stands, it is rather difficult to study mathematically the convergence of the resulting series of iterations. In fact, two difficulties arise: (i) the periodicity of $\epsilon(l, z)$ and the fact that the integration over $l^{\prime}$ extends over an infinite region (a typical feature when diffraction occurs); (ii) the presence of derivatives of $\bar{D}$ inside the integrals whenever $\bar{\nabla} \epsilon \neq 0$ (a direct consequence of Maxwell's equations and the previous assumptions). Accordingly, in order to cope with those difficulties, we shall perform in the next section two different transformations which will lead to a new integral equation for $\bar{D}(\bar{x}, \bar{K}, \lambda)$. The latter will enable one to study and establish rigorously the convergence of its corresponding series of iterations.

\section{The new integral equation and its convergence}

\subsection{The periodicity problem: first transformation}

Due to the periodicity of $\epsilon$ in the $\bar{a}_{1}-\bar{a}_{2}$ plane, one has

$$
\begin{aligned}
& \overline{\boldsymbol{D}}\left(\boldsymbol{l}+n_{1} a_{1}+n_{2} a_{2}, z, \overline{\boldsymbol{K}}, \lambda\right)=\exp \left[\mathrm{i} \boldsymbol{K} \cdot\left(n_{1} a_{1}+n_{2} a_{2}\right)\right] \overline{\boldsymbol{D}}(l, z, \overline{\boldsymbol{K}}, \lambda) \\
& n_{1}, n_{2}=0, \pm 1, \pm 2, \ldots
\end{aligned}
$$

In order to justify this property notice that: (i) if one looks for solutions of the wave equation (2.2) as a suitable superposition of plane waves, equation (3.1.1) is fulfilled by any term of the superposition; (ii) equation (3.1.1) is the proper generalisation of the well known Bloch theorem for electronic wavefunctions in crystals (Ziman 1964); (iii) if one considers the formal iterations of equation (2.4), replaces $G_{\alpha \beta}^{\mathrm{T}}$ by its Fourier integral representation (2.5) and $\epsilon$ by its Fourier series expansions and manipulates, 
equation (3.1.1) is seen to be verified by each iterate. Equation (3.1.1) will be fully consistent with all our later developments. Let $\Omega$ denote the finite region determined by $l^{\prime}=0, a_{1}, a_{2}, a_{1}+a_{2}$, in the $a_{1}-a_{2}$ plane.

By regarding the whole integration region for $l^{\prime}$ in equation (2.4) as the union of infinitely many copies of $\Omega$, using the periodicity of $\epsilon$ and equation (3.1.1), (2.4) can be transformed into:

$$
\begin{aligned}
& \overline{\boldsymbol{D}}(l, z, \overline{\boldsymbol{K}}, \lambda)=\overline{\boldsymbol{D}}_{0}(\overline{\boldsymbol{x}}, \overline{\boldsymbol{K}}, \lambda)+\int_{\Omega} \mathrm{d}^{2} l^{\prime} \int_{-\alpha / 2}^{+a / 2} \mathrm{~d} z^{\prime} \mathscr{S}^{\mathrm{T}}\left(l-l^{\prime}, z-z^{\prime}\right) \bar{j}\left(l^{\prime}, z^{\prime}\right) \\
& \mathscr{G}^{\mathrm{T}}=\left(\mathscr{G}_{\alpha \beta}^{\mathrm{T}}\right), \quad \mathscr{G}_{\alpha \beta}^{\mathrm{T}}(l, z)=\sum_{n_{1}, n_{2}=-\infty}^{+\infty} G_{\alpha \beta}^{\mathrm{T}}\left(l-\left(n_{1} a_{1}+n_{2} a_{2}\right), z\right) \exp \left[\mathrm{K} \cdot\left(n_{1} a_{1}+n_{2} a_{2}\right)\right] .
\end{aligned}
$$

A direct, although lengthy, calculation, where use is made of the Fourier integral representation for $G_{\alpha \beta}^{\mathrm{T}}$ (equation (2.5)), the Poisson sum formula (Morse and Feshbach 1953) and the residue theorem, leads to an alternative representation for $\mathscr{S}_{\alpha \beta}^{\mathrm{T}}$ (the structural transverse Green function):

$$
\mathscr{G}_{\alpha \beta}^{\mathrm{T}}(l, z)=\frac{1}{2 \pi K^{2} \Omega_{A}} \sum_{n_{1}, n_{2}=-\infty}^{+\infty} g_{\alpha \beta}\left(z, n_{1}, n_{2}\right) \exp \left[\mathrm{i} K\left(n_{1} n_{2}\right) . l\right]
$$

where, if $\bar{a}_{3}=(0,0,1)$ then:

$$
\begin{aligned}
& \bar{b}_{1}=2 \pi \frac{\bar{a}_{2} \times \bar{a}_{3}}{\Omega_{\mathrm{A}}}=\left(b_{1}, 0\right) \\
& \bar{b}_{2}=2 \pi \frac{\bar{a}_{3} \times \bar{a}_{1}}{\Omega_{A}}=\left(b_{2}, 0\right), \quad \Omega_{A}=\left(\bar{a}_{1} \times \bar{a}_{2}\right) \cdot \tilde{a}_{3}
\end{aligned}
$$

and

$$
\boldsymbol{K}\left(n_{1} n_{2}\right)=K+n_{1} b_{1}+n_{2} b_{2}=\left(K_{1}\left(n_{1} n_{2}\right), K_{2}\left(n_{1} n_{2}\right)\right)
$$

and on the other hand $(\alpha, \beta=1,2)$ :

$$
\begin{aligned}
g_{\alpha \beta}\left(z, n_{1} n_{2}\right)= & \pi \mathrm{i}\left[K_{\alpha}\left(n_{1} n_{2}\right) K_{\beta}\left(n_{1} n_{2}\right)-K^{2} \delta_{\alpha \beta}\right] \frac{\exp \left[\mathrm{i}|z|\left(K^{2}-K^{2}\left(n_{1} n_{2}\right)\right)^{1 / 2}\right]}{\left(K^{2}-K^{2}\left(n_{1} n_{2}\right)\right)^{1 / 2}} \\
& -\pi K_{\alpha}\left(n_{1} n_{2}\right) K_{\beta}\left(n_{1} n_{2}\right) \frac{\exp \left[-|z|\left(K^{2}\left(n_{1} n_{2}\right)\right)^{1 / 2}\right]}{\left(K^{2}\left(n_{1} n_{2}\right)\right)^{1 / 2}} \\
g_{3 \beta}\left(z, n_{1} n_{2}\right)= & g_{\beta 3}\left(z, n_{1} n_{2}\right) \\
= & \pi K_{\beta}\left(n_{1} n_{2}\right) \frac{\mathrm{d}}{\mathrm{d} z}\left(\frac{\exp \left[\mathrm{i}|z|\left(K^{2}-K^{2}\left(n_{1} n_{2}\right)\right)^{1 / 2}\right]}{\left(K^{2}-K^{2}\left(n_{1} n_{2}\right)\right)^{1 / 2}}+\mathrm{i} \frac{\exp \left[-|z|\left(K^{2}\left(n_{1} n_{2}\right)\right)^{1 / 2}\right]}{\left(K^{2}\left(n_{1} n_{2}\right)\right)^{1 / 2}}\right) \\
g_{33}\left(z, n_{1} n_{2}\right)= & -\mathrm{i} \pi K^{2}\left(n_{1} n_{2}\right)\left(\frac{\exp \left[\mathrm{i}|z|\left(K^{2}-K^{2}\left(n_{1} n_{2}\right)\right)^{1 / 2}\right]}{\left(K^{2}-K^{2}\left(n_{1} n_{2}\right)\right)^{1 / 2}}+\mathrm{i} \frac{\exp \left[-|z|\left(K^{2}\left(n_{1} n_{2}\right)\right)^{1 / 2}\right]}{\left(K^{2}\left(n_{1} n_{2}\right)\right)^{1 / 2}}\right) .
\end{aligned}
$$

Notice that equation (3.1.3) is useful for studying the short-distance behaviour of $\mathscr{G}_{\alpha \beta}^{\mathrm{T}}$ (as $(l, z) \rightarrow 0$, the term $n_{1}=n_{2}=0$ dominates), while the representation (3.1.4-5) is essential to obtain the long-distance behaviour of $\mathscr{G}_{\alpha \beta}^{\mathrm{T}}$, which, in turn, will lead us 
later to the diffraction amplitudes. The asymptotic behaviour of $\mathscr{G}_{\alpha \beta}^{\mathrm{T}}\left(l-l^{\prime}, z-z^{\prime}\right)$ as $z \rightarrow \pm \infty$ for fixed $l, l^{\prime}$ and $z^{\prime}$ is

$\mathscr{G}_{\alpha \beta}^{\mathrm{T}}\left(l-l^{\prime}, z-z^{\prime}\right)$

$$
\begin{aligned}
\approx & \sum_{\alpha(\boldsymbol{K}, K)} \frac{i}{2\left(K^{2}-K^{2}\left(n_{1} n_{2}\right)\right)^{1 / 2} \Omega_{A}} \exp \left\{i \left[K\left(n_{1} n_{2}\right)\left(l-l^{\prime}\right)\right.\right. \\
& \left.\left. \pm\left(K^{2}-K^{2}\left(n_{1} n_{2}\right)\right)^{1 / 2}\left(z-z^{\prime}\right)\right]\right\} \sum_{\lambda^{\prime}} D_{0}\left(\bar{K}_{ \pm}^{\prime}\left(n_{1} n_{2}\right) \lambda^{\prime}\right)_{\alpha} D_{0}^{*}\left(\bar{K}_{ \pm}^{\prime}\left(n_{1} n_{2}\right) \lambda^{\prime}\right)_{\beta} .
\end{aligned}
$$

Here $d(\boldsymbol{K}, \boldsymbol{K})$ denotes the finite set of pairs $\left(n_{1}, n_{2}\right)$ such that $K^{2} \geqslant K^{2}\left(n_{1} n_{2}\right)$ (as any pair $\left(n_{1}, n_{2}\right)$ not fulfilling this condition is associated with a contribution to (3.1.4) which vanishes exponentially).

$$
\overline{\mathbf{K}}_{ \pm}^{\prime}\left(n_{1} n_{2}\right)=\left(\boldsymbol{K}\left(n_{1} n_{2}\right), \pm\left(K^{2}-\boldsymbol{K}^{2}\left(n_{1} n_{2}\right)\right)^{1 / 2}\right)=\left(K_{ \pm}^{\prime}\left(n_{1} n_{2}\right)_{1}, K_{ \pm}^{\prime}\left(n_{1} n_{2}\right)_{2}, K_{ \pm}^{\prime}\left(n_{1} n_{2}\right)_{3}\right)
$$

and the complex polarisation vectors $\overline{\boldsymbol{D}}_{0}\left(\overline{\boldsymbol{K}}_{ \pm}^{\prime}\left(n_{1} n_{2}\right), \lambda^{\prime}\right)\left(\lambda^{\prime}\right.$ taking only two values) fulfill:

$$
\begin{aligned}
& \sum_{\lambda^{\prime}} D_{0}\left(\overline{\boldsymbol{K}}_{ \pm}^{\prime}\left(n_{1} n_{2}\right), \lambda^{\prime}\right)_{\alpha} D_{0}^{*}\left(\overline{\boldsymbol{K}}_{ \pm}^{\prime}\left(n_{1} n_{2}\right), \lambda^{\prime}\right)_{\beta}=\delta_{\alpha \beta}-\frac{K_{ \pm}^{\prime}\left(n_{1} n_{2}\right)_{\alpha} K_{ \pm}^{\prime}\left(n_{1} n_{2}\right)_{\beta}}{K^{2}} \\
& \overline{\boldsymbol{K}}_{ \pm}^{\prime}\left(n_{1} n_{2}\right) \cdot \overline{\boldsymbol{D}}_{0}\left(\overline{\boldsymbol{K}}_{ \pm}^{\prime}\left(n_{1} n_{2}\right), \lambda^{\prime}\right)=0, \quad \overline{\boldsymbol{D}}_{0}\left(\overline{\boldsymbol{K}}_{+}^{\prime}(00), \lambda\right)=\overline{\boldsymbol{D}}_{0}(\overline{\boldsymbol{K}}, \lambda) .
\end{aligned}
$$

\subsection{Elimination of derivatives: second transformation}

The second transformation leads from equation (3.1.2), which still contains derivatives of $\overline{\boldsymbol{D}}$, to the final integral equation, which is free of them. For that purpose: (i) one recalls equation (2.3) and appeals to standard vector identities in order to replace derivatives of $\overline{\boldsymbol{D}}$ by derivatives of $\boldsymbol{A}$ and $\mathscr{S}_{\alpha \beta}^{\mathrm{T}}$ plus certain divergences; (ii) one uses Green's identities for the finite three-dimensional integration region appearing in equation (3.1.2) in order to transform those divergences into surface terms; (iii) one realises that all surface terms cancel due to the periodicity of $\epsilon$, equation (3.1.1) and a similar property for $\mathscr{G}_{\alpha \beta}^{\mathrm{T}}$. The final integral equation for $\overline{\boldsymbol{D}}=\left(D_{1}, D_{2}, D_{3}\right)$ reads

$$
\begin{aligned}
D_{\alpha}(l, z, \bar{K}, \lambda) & =D_{0}(\bar{x}, \overline{\mathbf{K}}, \lambda)_{\alpha} \\
& +\int_{\Omega} \mathrm{d}^{2} l^{\prime} \int_{-a / 2}^{+a / 2} \mathrm{~d} z^{\prime} \sum_{\beta=1}^{3} U_{\alpha \beta}\left(l-l^{\prime}, z-z^{\prime}\right) D_{\alpha}\left(l^{\prime}, z^{\prime}, \bar{K}, \lambda\right), \quad \alpha=1,2,3
\end{aligned}
$$

where $\left(\bar{x}^{\prime}=\left(l^{\prime}, z^{\prime}\right)\right)$

$$
\begin{aligned}
& U_{\alpha \beta}\left(l-l^{\prime}, z-z^{\prime}\right) \\
&= K^{2}\left(1-\frac{\epsilon\left(l^{\prime}, z^{\prime}\right)}{\epsilon_{0}}\right) \mathscr{G}_{\alpha \beta}^{\mathrm{T}}\left(l-l^{\prime}, z-z^{\prime}\right) \\
&+\sum_{\gamma, \sigma=1}^{3}\left[\bar{\nabla}_{z^{\prime}} \times \bar{B}_{\alpha}\left(l, z ; l^{\prime}, z^{\prime}\right)\right]_{\gamma} \epsilon_{\gamma \sigma \beta}\left(\bar{\nabla}_{z} \frac{1}{\epsilon\left(l^{\prime}, z^{\prime}\right)}\right)_{\sigma} \\
&+\left[\bar{\nabla}_{z^{\prime}} \times \overline{\boldsymbol{C}}_{\alpha}\left(l, z ; l^{\prime}, z^{\prime}\right)\right]_{\beta}
\end{aligned}
$$




$$
\begin{aligned}
\bar{B}_{\alpha}=\left(B_{\alpha 1}, B_{\alpha 2}, B_{\alpha 3}\right), \quad B_{\alpha \beta}\left(l, z ; l^{\prime}, z^{\prime}\right)=\mathscr{G}_{\alpha \beta}^{\mathrm{T}}\left(l-l^{\prime}, z-z^{\prime}\right) \epsilon\left(l^{\prime}, z^{\prime}\right) \\
\bar{C}_{\alpha}=\left(C_{\alpha 1}, C_{\alpha 2}, C_{\alpha 3}\right), \\
C_{\alpha \beta}\left(l, z ; l^{\prime}, z^{\prime}\right)=-\sum_{\gamma, \sigma=1}^{3} \mathscr{G}_{\alpha \beta}^{\mathrm{T}}\left(l-l^{\prime}, z-z^{\prime}\right) \epsilon_{\gamma \sigma \beta}\left[\bar{\nabla}_{\bar{z}^{\prime}}\left(\ln \epsilon\left(l^{\prime}, z^{\prime}\right)\right)\right]_{\sigma}
\end{aligned}
$$

Here $\epsilon_{\gamma \sigma \beta}$ is the usual totally antisymmetric tensor with three indices $\left(\epsilon_{123}=+1\right)$.

\subsection{Convergence of iterations}

Upon iterating equation (3.2.1) one finds:

$$
\begin{gathered}
D_{\alpha}(\boldsymbol{l}, z, \overline{\boldsymbol{K}}, \lambda)=\sum_{n=0}^{\infty} D_{n}(\boldsymbol{l}, z, \overline{\boldsymbol{K}}, \lambda)_{\alpha} \\
D_{n}(\boldsymbol{l}, z, \overline{\boldsymbol{K}}, \lambda)_{\alpha}=\int_{\Omega} \mathrm{d}^{2} \boldsymbol{l}^{\prime} \int_{-\alpha / 2}^{+\alpha / 2} \mathrm{~d} z^{\prime} \sum_{\beta=1}^{3} U_{\alpha \beta}\left(\boldsymbol{l}-\boldsymbol{l}^{\prime}, z-z^{\prime}\right) D_{n-1}\left(\boldsymbol{l}^{\prime}, \boldsymbol{z}^{\prime}, \overline{\boldsymbol{K}}, \lambda\right)_{\beta} .
\end{gathered}
$$

Let us introduce, as $l$ varies in $\Omega, z$ in $(-\infty,+\infty)$ and $\alpha=1,2,3$

$$
u=\max \int_{\Omega} \mathrm{d}^{2} l^{\prime} \int_{-a / 2}^{+a / 2} \mathrm{~d} z^{\prime} \sum_{\beta=1}^{3}\left|U_{\alpha \beta}\left(l-l^{\prime}, z-z^{\prime}\right)\right| .
$$

Then, if $u<1$, the series (3.3.1) converges. In fact, majorising one gets:

$$
\max _{\substack{\boldsymbol{l} \in \boldsymbol{\alpha} \\-\infty<<<+\infty \\ \alpha=1,2,3}}\left|D_{n}(l, z, \overline{\boldsymbol{K}}, \lambda)_{\alpha}\right| \leqslant u\left(\max _{\substack{l^{\prime} \in \boldsymbol{R} \\-\infty<z^{\prime}<+\infty \\ \beta=1,2,3}}\left|D_{n-1}\left(l^{\prime}, z^{\prime}, \overline{\boldsymbol{K}}, \lambda\right)_{\beta}\right|\right)
$$

which, summing the resulting geometric series, leads to

$$
\max _{\substack{l \in \Omega \\-\infty<z<+\infty \\ \alpha=1,2,3}}\left|D_{\alpha}(l, z, \overline{\boldsymbol{K}}, \lambda)\right| \leqslant \frac{1}{1-u}\left(\max _{\alpha=1,2,3}\left|D_{0}(\overline{\boldsymbol{K}}, \lambda)_{\alpha}\right|\right)
$$

so that (3.3.1) converges and satisfies equation (3.2.1). It is important to realise that $u$ is always finite, due to the use of $G_{\alpha \beta}^{\mathrm{T}}$, and provided that $K^{2} \neq \boldsymbol{K}^{2}\left(n_{1} n_{2}\right)$ for any $\left(n_{1}, n_{2}\right)$ and that $\epsilon$ be physically reasonable (both $\bar{\nabla}(1 / \epsilon)$ and $\bar{\nabla}(\ln \epsilon)$ should be finite). This property can be proved as follows.

(i) as $(l, z) \rightarrow\left(l^{\prime}, z^{\prime}\right)$ the behaviour of $G_{\alpha \beta}^{\mathrm{T}}\left(l-l^{\prime}, z-z^{\prime}\right)$ given after equation (2.5) implies through equations (3.2.2-4) that any contribution to $U_{\alpha \beta}\left(l-l^{\prime}, z-z^{\prime}\right)$ diverges, at most, as $1 /\left[\left(l-l^{\prime}\right)^{2}+\left(z-z^{\prime}\right)^{2}\right]$ which gives rise to a finite integral in equation (3.3.2); (ii) for large $|z|$, the integral in equation (3.3.2) is finite, since equation (3.1.6) contains only a finite number of contributions. Had we used the customary Green function $\Gamma_{\alpha \beta}$ instead of $G_{\alpha \beta}^{\mathrm{T}}$, then an analogous study would lead to an equation similar to equation (3.2.1), with a new kernel $U_{\alpha \beta}$ which diverges as $1 /\left[\left(\boldsymbol{l}-\boldsymbol{l}^{\prime}\right)^{2}+\left(z-z^{\prime}\right)^{2}\right]^{2}$ as $(\boldsymbol{l}, z) \rightarrow\left(\boldsymbol{l}^{\prime}, z^{\prime}\right)$, and thus forces the new integral, analogous to that in equation (3.3.2) to diverge. Thus a convergence study in terms of $\Gamma_{\alpha \beta}$ is not possible, due to its stronger divergence as $|\bar{x}| \rightarrow 0$. 


\section{Transmission and reflection amplitudes}

We shall study the asymptotic behaviour of $\overline{\boldsymbol{D}}(\boldsymbol{l}, z, \overline{\boldsymbol{K}}, \lambda)$ as $z \rightarrow \pm \infty$ for fixed $\boldsymbol{l}$. Although for the sake of rigour one should use equation (3.2.1), as far as the diffraction amplitudes $T_{ \pm}\left(n_{1}, n_{2}, l^{\prime}\right)$ are concerned, it is simpler (and completely equivalent) to start with equation (3.1.2) and to perform later suitable partial integrations di.ectly in $T_{ \pm}\left(n_{1} n_{2}\right)$, in order to cope with the derivatives of $\overline{\boldsymbol{D}}$. Equation (3.1.6) leads to

$$
\begin{aligned}
\overline{\boldsymbol{D}}(\boldsymbol{l}, z, \overline{\boldsymbol{K}}, \lambda) \simeq & \sum_{d(\boldsymbol{K} \boldsymbol{K})} \sum_{\lambda^{\prime}} \exp \left[\mathrm{i} \overline{\boldsymbol{K}}_{ \pm}^{\prime} \overline{\boldsymbol{x}}\right] \cdot T_{ \pm}\left(n_{1}, n_{2}, \lambda^{\prime}\right) \cdot \overline{\boldsymbol{D}}_{0}\left(\overline{\boldsymbol{K}}_{ \pm}^{\prime}\left(n_{1} n_{2}\right), \lambda^{\prime}\right) \quad z \rightarrow \pm \infty \\
T_{ \pm}\left(n_{1}, n_{2}, \lambda^{\prime}\right)= & \delta_{ \pm}\left(n_{1} n_{2}\right) \delta_{\lambda^{\prime} \lambda}+\frac{\mathrm{i}}{2\left(\boldsymbol{K}^{2}-\boldsymbol{K}^{2}\left(n_{1} n_{2}\right)\right)^{1 / 2} \Omega_{A}} \\
& \times \int_{\Omega} \mathrm{d}^{2} \boldsymbol{l}^{\prime} \int_{-a / 2}^{+a / 2} \mathrm{~d} z^{\prime} \exp \left(-\mathrm{i} \overline{\boldsymbol{K}}_{ \pm}^{\prime}\left(n_{1} n_{2}\right) \overline{\boldsymbol{x}}^{\prime}\right) \cdot \overline{\boldsymbol{D}}_{0}^{*}\left(\overline{\boldsymbol{K}}_{ \pm}^{\prime}\left(n_{1} n_{2}\right) \lambda^{\prime}\right) \bar{j}\left(\boldsymbol{l}^{\prime}, z^{\prime}\right)
\end{aligned}
$$

where $\delta_{-}\left(n_{1} n_{2}\right)=0$, for any $\left(n_{1}, n_{2}\right), \delta_{+}(00)=1$ and $\delta_{+}\left(n_{1} n_{2}\right)=0$ for $\left(n_{1}, n_{2}\right) \neq(0,0)$.

$T_{+}$and $T_{-}$are the transmission and reflection amplitudes, respectively. We shall point out that when $K$ is such that $K^{2}=K^{2}\left(n_{1} n_{2}\right)$, new diffracted waves are created: then, the convergence condition $u<1$ breaks down although the diffraction amplitudes remain finite.

The usual Debye-Born-Rayleigh-Gans (DBRG) approximation $T_{ \pm}\left(n_{1}, n_{2}, l^{\prime}\right)_{\text {DBRG }}$ is obtained upon replacing $\overline{\boldsymbol{D}}\left(\boldsymbol{l}^{\prime}, z^{\prime}, \overline{\boldsymbol{K}}, \lambda\right)$ by $\overline{\boldsymbol{D}}_{0}\left(\overline{\boldsymbol{x}}^{\prime}, \overline{\boldsymbol{K}}, \lambda\right)$ inside the integral in equation (4.2) (this is what is done by Landau and Lifchitz 1969) and an important problem consists in giving bounds for the error of the approximation $T_{ \pm}\left(n_{1}, n_{2}, l^{\prime}\right) \simeq$ $T_{ \pm}\left(n_{1}, n_{2}, l^{\prime}\right)_{\text {DBRG. }}$. For this purpose, derivatives of $\bar{D}$ inside the integrals in equation (4.2) are again difficult to handle and a transformation similar to that leading from equation (3.1.2) to equation (3.2.1) has to be performed. A suitable Green identity together with the subsequent cancellation of surface terms due to periodicity and equation (3.1.1), yields after some calculations:

$T_{ \pm}\left(n_{1}, n_{2}, \lambda^{\prime}\right)-T_{ \pm}\left(n_{1}, n_{2}, \lambda^{\prime}\right)_{\mathrm{DBRG}}$

$$
=\int_{\Omega} \mathrm{d}^{2} \boldsymbol{l}^{\prime} \int_{-a / 2}^{+a / 2} \mathrm{~d} z^{\prime} \overline{\boldsymbol{W}}_{ \pm}\left(\boldsymbol{l}^{\prime}, z^{\prime}, n_{1}, n_{2}, \lambda^{\prime}\right)\left[\boldsymbol{D}\left(\boldsymbol{l}^{\prime} z^{\prime} \overline{\boldsymbol{K}} \lambda\right)-\overline{\boldsymbol{D}}_{0}\left(\overline{\boldsymbol{x}}^{\prime} \overline{\boldsymbol{K}} \lambda\right)\right]
$$

where

$$
\begin{aligned}
& \bar{W}_{ \pm}\left(\boldsymbol{l}^{\prime}, z^{\prime}, n_{1}, n_{2}, \lambda^{\prime}\right) \\
&=\frac{\mathrm{i}}{2\left[\boldsymbol{K}^{2}-\boldsymbol{K}^{2}\left(n_{1} n_{2}\right)\right]^{1 / 2} \Omega_{A}}\left[\exp \left(-\mathrm{i} \overline{\boldsymbol{K}}_{ \pm}^{\prime}\left(n_{1} n_{2}\right) \overline{\boldsymbol{x}}^{\prime}\right)\right. \\
& \times \boldsymbol{K}^{2}\left(1-\frac{\epsilon\left(\boldsymbol{l}^{\prime}, z^{\prime}\right)}{\epsilon_{0}}\right) \overline{\boldsymbol{D}}_{0}^{*}\left(\overline{\boldsymbol{K}}_{ \pm}^{\prime}\left(n_{1} n_{2}\right) \lambda^{\prime}\right) \\
&-\bar{\nabla}_{\bar{x}^{\prime}} \times\left[\exp \left(-\mathrm{i} \overline{\boldsymbol{K}}_{ \pm}^{\prime}\left(n_{1} n_{2}\right) \overline{\boldsymbol{x}}^{\prime}\right) \overline{\boldsymbol{D}}_{0}^{*}\left(\overline{\boldsymbol{K}}_{ \pm}^{\prime}\left(n_{1} n_{2}\right) \lambda^{\prime}\right) \times\left(\bar{\nabla}_{\overline{\boldsymbol{x}}^{\prime}} \ln \epsilon\left(\boldsymbol{l}^{\prime}, z^{\prime}\right)\right)\right] \\
&\left.+\left\{\overline{\boldsymbol{\nabla}}_{\bar{x}^{\prime}} \times\left[\exp \left(-\mathrm{i} \overline{\boldsymbol{K}}_{ \pm}^{\prime}\left(n_{1} n_{2}\right) \overline{\boldsymbol{x}}^{\prime}\right) \overline{\boldsymbol{D}}_{0}^{*}\left(\overline{\boldsymbol{K}}_{ \pm}^{\prime}\left(n_{1} n_{2}\right) \lambda^{\prime}\right) \epsilon\left(\boldsymbol{l}^{\prime}, z^{\prime}\right)\right]\right\} \times\left(\overline{\boldsymbol{\nabla}}_{\bar{x}^{\prime}} \frac{1}{\epsilon\left(l^{\prime}, z^{\prime}\right)}\right)\right]
\end{aligned}
$$


Define:

$$
a_{ \pm}\left(n_{1}, n_{2}, \lambda^{\prime}\right)=\int_{\Omega} \mathrm{d}^{2} l^{\prime} \int_{-a / 2}^{+a / 2} \mathrm{~d} z^{\prime}\left|\bar{W}_{ \pm}\left(l^{\prime}, z^{\prime}, n_{1}, n_{2}, \lambda^{\prime}\right)\right|
$$

Then, some majorisations, similar to those in $\$ 3.3$, lead to the desired bounds

$$
\left|T_{ \pm}\left(n_{1}, n_{2}, \lambda^{\prime}\right)-T_{ \pm}\left(n_{1}, n_{2}, \lambda^{\prime}\right)_{\mathrm{DBRG}}\right| \leqslant a_{ \pm}\left(n_{1}, n_{2}, \lambda^{\prime}\right) \frac{u}{1-u}\left(\max _{\alpha=1,2,3}\left|D_{0}(\overline{\mathbf{K}}, \lambda)_{\alpha}\right|\right) .
$$

\section{Estimates of the convergence condition}

We shall discuss here the convergence condition $u<1$ under the following assumptions.

(1) $\left|a_{1}\right|$ and $\left|a_{2}\right|$ are of the same order of magnitude, with $a_{1} \cdot a_{2}=0$, and $a=$ $M_{1}\left|a_{1}\right|=M_{2}\left|a_{2}\right|$, both $M_{1}$ and $M_{2}$ being large dimensionless numbers.

(2) The wavelength is of the order of the lattice periods, $K=0$, and $K^{2}-$ $\left(m_{1} b_{1}+m_{2} b_{2}\right)^{2} \neq 0$ for any $m_{1}, m_{2}=0, \pm 1 \ldots$ For later application, let $K$ be close to $\frac{1}{2} n_{1}\left|b_{1}\right|$ or $\frac{1}{2} n_{2}\left|b_{2}\right|$, where $n_{1}, n_{2}$ are small integers (say, $1 \leqslant n_{1}, n_{2} \leqslant 3$ ). Then, the set $d(0, K)$ contains only a small number of diffracted 'beams'.

(3) Let

$$
\epsilon(l, z)=\epsilon_{0}\left(1+\Delta \cos \left[\left(\sigma_{1} b_{1}+\sigma_{2} b_{2}\right) \cdot l+b_{3} z\right]\right)
$$

for $-a / 2<z<+a / 2$ where $\Delta$ is constant and very small compared to $1, \sigma_{1}$ and $\sigma_{2}$ are small integers (say, $\sigma_{1}, \sigma_{2}=0,1,2$ ) and $b_{3}$ is, roughly, of the same order as $\sigma_{1}\left|b_{1}\right|$ or $\sigma_{2}\left|\boldsymbol{b}_{2}\right|$.

When $\left|a_{1}\right|,\left|a_{2}\right|=1 \AA$, these assumptions correspond qualitatively and even quantitatively to $x$-ray diffraction by thin crystals (see references in $\$ 1$ ).

It is hard to evaluate $u$ exactly. Fortunately, by virtue of the above assumptions, the dominant contributions to $u$ and, thus, an order of magnitude estimate for it, can be obtained by replacing $\mathscr{G}_{\alpha \beta}^{\mathrm{T}}\left(I-l^{\prime}, z-z^{\prime}\right)$ in equations (3.3.2) and (3.2.2-4) by its asymptotic form (3.1.6), so that only the pairs $\left(n_{1}, n_{2}\right)$ belonging to $d(0, K)$ (a small set) contribute. In order to justify this recipe, one decomposes the full integration domain in equation (3.3.2) into the following regions: (i) a sphere of centre $(l, z)$ and radius $\delta$ chosen as a small fraction of $\left|a_{1}\right|,\left|a_{2}\right|$; (ii) the two 'big slices' formed by all $\left(l^{\prime}, z^{\prime}\right)$ such that $l^{\prime}$ belongs to $\Omega$ and either $-a / 2<z^{\prime}<z-\delta$ or $z+\delta<z^{\prime}<+a / 2$; (iii) the remainder. Using the expansion (3.1.3) in region (i), equation (3.1.4) in (ii) and both judiciously for estimates in (iii), one can see that for $\delta<\left|a_{1}\right|,\left|a_{2}\right| \ll a$, the dominant contribution to $u$ comes precisely from region (ii) and that, moreover, the latter can be estimated by the asymptotic form (3.1.6) in the full integration domain.

By using (3.3.2), (3.2.2-4) and (3.1.6), some calculations lead to the following approximation for $u$ :

$$
\begin{aligned}
u \leqslant a \Delta \max _{\alpha=1,2,3} & {\left[\sum_{d(0, K)} \sum_{\beta=1}^{3} \frac{\left|\delta_{\alpha \beta}-K^{-2}\left(K_{ \pm}^{\prime}\left(n_{1} n_{2}\right)_{\alpha} K_{ \pm}^{\prime}\left(n_{1} n_{2}\right)_{\beta}\right)\right|}{2\left(K^{2}-K^{2}\left(n_{1}, n_{2}\right)\right)^{1 / 2}}\right.} \\
& \left.\times\left(w_{\beta}+\sum_{\gamma=1}^{3} w_{\beta \gamma}\left|K_{ \pm}^{\prime}\left(n_{1} n_{2}\right)_{\gamma}\right|\right)\right]
\end{aligned}
$$


where one should take the largest value out of the possibilities + and - , higher-order terms in $\Delta$ have been neglected and

$$
\begin{gathered}
w_{1}=K^{2}+\left(\frac{2 \pi \sigma_{2}}{\left|a_{2}\right|}\right)^{2}+b_{3}^{2}+\frac{2 \pi \sigma_{1}}{\left|a_{1}\right|}\left(\frac{2 \pi \sigma_{2}}{\left|a_{2}\right|}+b_{3}\right) \\
w_{2}=K^{2}+b_{3}^{2}+\left(\frac{2 \pi \sigma_{1}}{\left|a_{1}\right|}\right)^{2}+\frac{2 \pi \sigma_{2}}{\left|a_{2}\right|}\left(b_{3}+\frac{2 \pi \sigma_{2}}{\left|a_{1}\right|}\right) \\
w_{3}=K^{2}+\left(\frac{2 \pi \sigma_{1}}{\left|a_{1}\right|}\right)^{2}+\left(\frac{2 \pi \sigma_{2}}{\left|a_{2}\right|}\right)^{2}+b_{3}\left(\frac{2 \pi \sigma_{1}}{\left|a_{1}\right|}+\frac{2 \pi \sigma_{1}}{\left|a_{2}\right|}\right) \\
w_{11}=\frac{2 \pi \sigma_{2}}{\left|a_{2}\right|}+b_{3}, \quad w_{12}=\frac{2 \pi \sigma_{1}}{\left|a_{1}\right|}+2 \frac{2 \pi \sigma_{2}}{\left|a_{2}\right|}, \quad w_{13}=\frac{2 \pi \sigma_{1}}{\left|a_{1}\right|}+2 b_{3} \\
w_{21}=\frac{2 \pi \sigma_{2}}{\left|a_{2}\right|}+2 \frac{2 \pi \sigma_{1}}{\left|a_{1}\right|}, \quad w_{22}=b_{3}+\frac{2 \pi \sigma_{1}}{\left|a_{1}\right|}, \quad w_{23}=\frac{2 \pi \sigma_{2}}{\left|a_{2}\right|}+2 b_{3} \\
w_{31}=b_{3}+2 \frac{2 \pi \sigma_{1}}{\left|a_{1}\right|}, \quad w_{32}=b_{3}+2 \frac{2 \pi \sigma_{2}}{\left|a_{2}\right|}, \quad w_{33}=\frac{2 \pi \sigma_{1}}{\left|a_{1}\right|}+\frac{2 \pi \sigma_{2}}{\left|a_{2}\right|} .
\end{gathered}
$$

By virtue of the above assumptions, the order of magnitude of $u$ is determined essentially by $a \Delta$, that is, by $M_{1} \Delta, M_{2} \Delta$, since the remaining terms in (5.1) will not modify it appreciably. For $\mathrm{x}$-rays in crystals, $\Delta$ is usually of order $10^{-5}-10^{-6}$ (Betterman and Cole 1964, Ewald 1965, Slater 1967) so that the series (3.3.1) converges for large values of $M_{1}, M_{2}$ up to, say $10^{4}$ or $10^{5}$ (that is, a slab thickness up to about $10^{-3} \mathrm{~cm}$ ). For sensibly larger values of $M_{1}, M_{2}$, the convergence of (3.3.1) may still hold, but it can not be warranted from our estimates.

When the condition $u<1$ holds, a bound for the absolute error of the usual DBRG approximation (Landau and Lifchitz 1969) can be estimated by using equations (4.5), (5.1) and the following bound $\left(\left(n_{1}, n_{2}\right)\right.$ belonging to $\left.d(0, K)\right)$ :

$$
\begin{aligned}
a_{ \pm}\left(n_{1}, n_{2}, \lambda^{\prime}\right) & \leqslant \frac{a \Delta\left|\overline{\boldsymbol{D}}_{0}^{*}\left(\overline{\boldsymbol{K}}_{ \pm}^{\prime}\left(n_{1} n_{2}\right) \lambda^{\prime}\right)\right|}{2\left(K^{2}-\boldsymbol{K}^{2}\left(n_{1} n_{2}\right)\right)^{1 / 2}}\left\{K^{2}+2\left[\sum_{\gamma=1}^{3}\left(w_{\gamma}-K^{2}\right)\right.\right. \\
& \left.\left.+\sum_{\gamma=1}^{3}\left|K_{ \pm}^{\prime}\left(n_{1} n_{2}\right)_{\gamma}\right|\left(\sum_{\substack{\alpha=1 \\
\alpha \neq \gamma}}^{3} w_{\alpha \alpha}\right)\right]\right\} .
\end{aligned}
$$

In equation (5.3), higher-order terms in $\Delta$ have been neglected and the order of magnitude is determined by $M_{1} \Delta, M_{2} \Delta$ again. Notice that an estimate for the relative error of the DBRG approximation is simply $u /(1-u)$. Thus, even if $u<1$ and the series (3.3.1) converges, this relative error may not be small when $M_{1} \Delta, M_{2} \Delta$ are close to 1 , that is, for not too small thickness. Hence, the corrections to the DBRG approximation arising from higher-order terms in (3.3.1) may be required.

\section{Acknowledgment}

This work is based upon a PhD Thesis presented by M L Calvo at the University of Madrid. We thank Professor A Durán for his direction and encouragement and Professor J Solana for a critical reading of the manuscript. 


\section{References}

Batterman B W and Cole H 1964 Rev. Mod. Phys. 36631

Born M and Wolf E 1965 Principles of Optics 3rd edn (Oxford: Pergamon)

Calvo M L and Durán A 1975 Nuovo Cim. 29B 277

Ewald P P 1965 Rev. Mod. Phys. 3746

James R W 1963 The Dynamical Theory of X-ray Diffraction: Solid State Physics vol. 15 eds H Ehrenreich, F Seitz and D Turnbull (New York: Academic)

Landau L and Lifchitz E 1969 Electrodynamique des Milieux continus (Moscow: Editions Mir) chap. 15

Morse P M and Feshbach H 1953 Methods of Theoretical Physics vol. 1 (New York: McGraw-Hill) chap. 4 Newton R G 1966 Scattering Theory of Waves and Particles (New York: McGraw-Hill) chap. 3

Slater J C 1967 Quantum Theory of Molecules and Solids vol. 3 (New York: McGraw-Hill) chap. 6

Vienot J C, Smigielski P and Royer H 1971 Holographie Optique (Developpements, Applications) (Paris: Dunod)

Zachariasen W H 1945 Theory of X-ray Diffraction in Crystals (New York: John Wiley)

Ziman J M 1964 Principles of the Theory of Solids (London: Cambridge University Press) chap. 1 